\title{
Differences between Algorithms for Spatial Interpolation of 3D Echocardiography Images
}

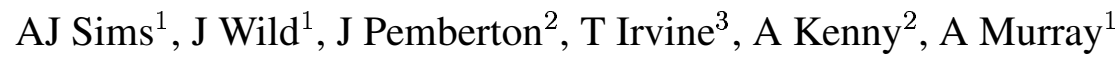 \\ ${ }^{1}$ Regional Medical Physics Department, Freeman Hospital, Newcastle upon Tyne, UK \\ ${ }^{2}$ Department of Cardiology, Freeman Hospital, Newcastle upon Tyne, UK \\ ${ }^{3}$ Department of Cardiology, Royal Victoria Infirmary, Newcastle upon Tyne, UK
}

\begin{abstract}
In 3D, ultrasound systems sample a conical volume of space with a given angular resolution, resulting in sparsely sampled regions far from the transducer. Image analysis operators require that each voxel of a cartesian volume has a value, and some form of interpolation is necessary.

$3 D$ images of the left ventricle ( $L V)$ of 10 patients were constructed from sequences of conventional B-mode frames which were successively rotated about the beam axis. Three different methods of interpolation were used to assign values to unsampled voxels. Reconstructed LV short axis views were extracted from the $3 D$ images and analysed using standard edge detection and threshold operators. Results from the image analysis were used to compare the three different methods of interpolation.

Results of this preliminary study show that the choice of interpolation method influences the results of image analysis operations.
\end{abstract}

\section{Introduction}

Quantitative three dimensional echocardiography is an emerging clinical tool with which important cardiac indicators, such as left ventricular volume (eg [1]) and ejection fraction can be measured. However, the quantity of information generated by three dimensional studies cannot easily be assimilated by human observers, and automatic, or semi-automatic image analysis methods are needed.

Tissue volume mapping by ultrasound is achieved by mechanical rotation of a 1D linear transducer array or by electronic beam steering with a $2 \mathrm{D}$ array to generate a sequence of A-mode scans which occupy a conical region of space. The process of assigning pixels from polar Amode scans to a $3 \mathrm{D}$ cartesian image leaves many voxels unassigned and interpolation is necessary.

Interpolation has usually been studied in the context of image resampling. Several authors [2, 3, 4] have compared different interpolation techniques for resampling images in $2 \mathrm{D}$, and shown that the optimal method depends upon the

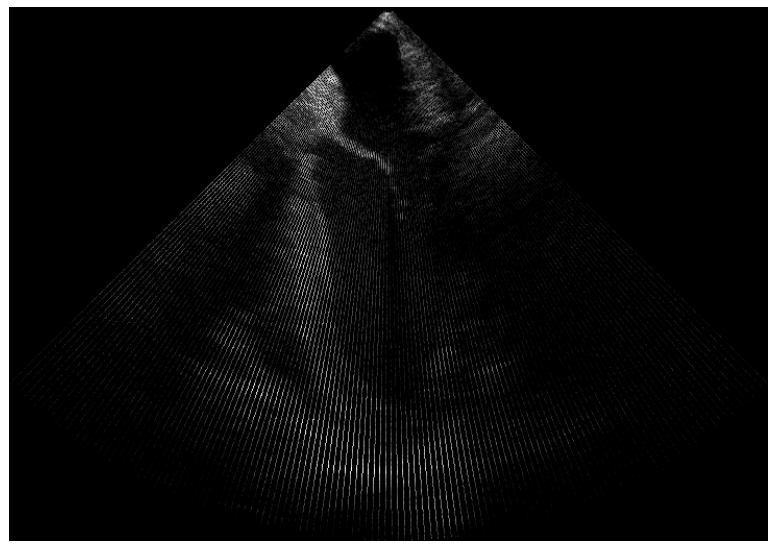

Figure 1. Uninterpolated LV long axis view of patient 2. Moiré fringes are an artefact of image reproduction.

nature of the source image. In 3D, shape-based methods have been shown [5] to out-perform gray level interpolation for resampling of MR and CT images. Where it is not possible to use a priori knowledge of image content, the choice of interpolation method in 3D has been shown to affect calculation of volume [6] and registration [7].

In this paper, we study the effect of different 3D interpolation techniques for cardiac ultrasound images, on the result of a common set of image analysis operations (edge detection, thresholding, binary erosion).

\section{Methods}

\subsection{Image acquisition}

Ten patients who had undergone coronary artery bypass graft surgery and who had impaired LV function were examined by trans-oesophageal cardiac ultrasound. Images were acquired with an ATL HDI 5000 ultrasound system with a $7 \mathrm{MHz}$ rotating trans-oesophageal probe (MPT 7-4) whilst ventilation was suspended. A sequence of frames over one cardiac cycle was captured for each orientation of 

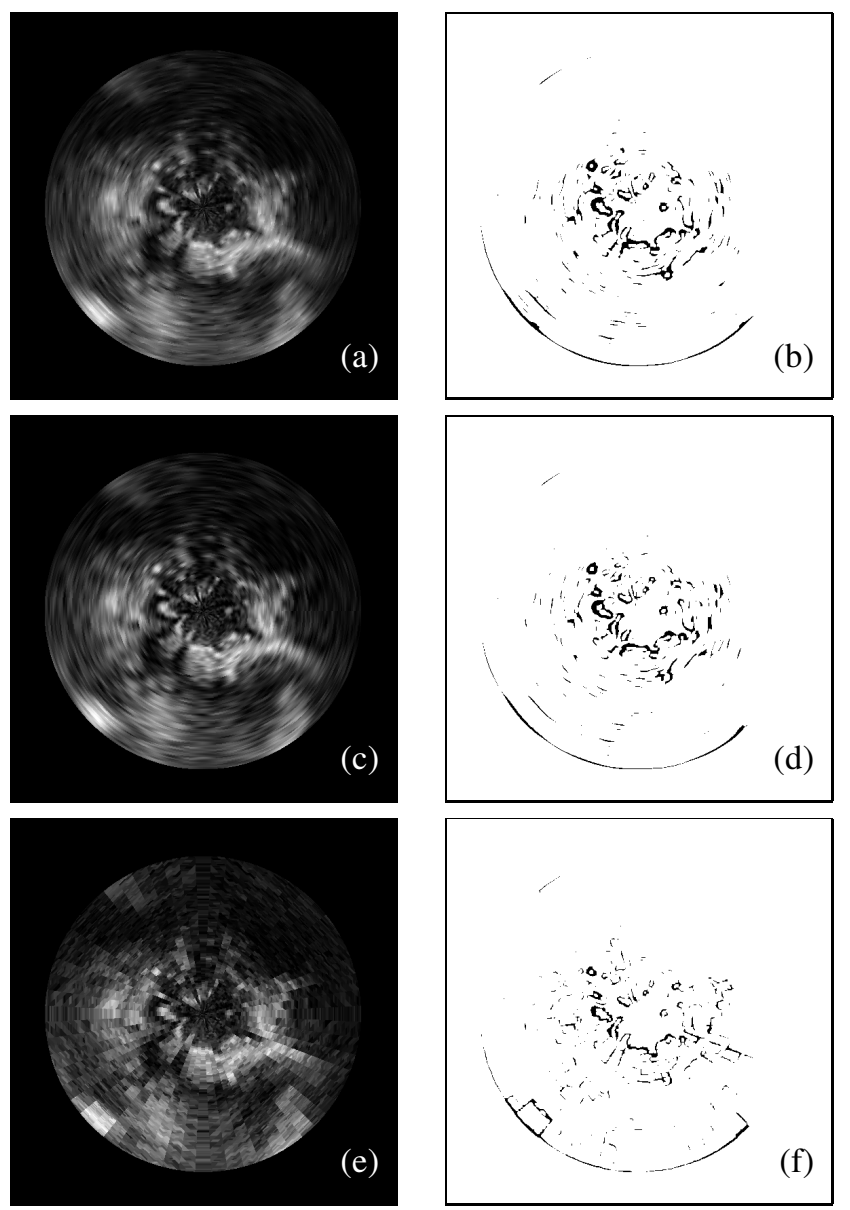

Figure 2. The effect of interpolation is shown by this LV short axis view for patient 2, reconstructed from a 3D image at end systole using three different methods of interpolation:- (a) reconstructed image using 1D linear interpolation; (b) corresponding binary edge map, after edge detection and thresholding; $(\mathrm{c}, \mathrm{d})$ as above but with 1D spline interpolation; (e,f) as above but with $3 \mathrm{D}$ nearest neighbour interpolation.

the transducer. Each A-mode scan line consisted of 512 linear pixels, and there were 128 scan lines in each frame, spaced at $0.71^{\circ}$ to cover a $90^{\circ}$ arc.

The image plane was successively rotated at $6^{\circ}$ intervals about an axis approximately parallel to the the LV long axis. The number of planes per R-R interval was constant for each patient and depended upon heart rate (range 12-18).

\subsection{Image processing}

Images were extracted from the ultrasound machine via raw, proprietary format files which contained scan sequences stored as A-mode images, together with machine settings for beam depth and beam angle. These files contained only sampled values, and no interpolation by the ultrasound system was used.

Specially written software was used to create 3D images from A-mode scans. Echo intensities in A-mode scans relating to a particular phase of the cardiac cycle were assigned to voxels in a 3D image according to their computed geometrical location in a $3 \mathrm{D}$ volume surrounding the left ventricle. Figure 1 shows a reconstructed 2D image, extracted from a 3D image of patient 2 at end systole. Only the sampled points are shown, no interpolation has been applied.

\subsection{Image interpolation}

After pixels from A-mode scans were assigned to voxels in 3D images, unassigned voxels that lay within the conical ultrasound beam volume were given an intensity value based on one of three interpolation techniques:-

$1 D$ linear: Unsampled voxels were assigned a value based on a linear interpolation of adjacent sampled pixels in a circular arc. The interpolation was first applied in planes of the original acquisition to fill in pixels along arcs of equal distance from the source, and then applied in planes perpendicular to the beam axis, along arcs of equal radial distance.

1D spline: As 1D linear, but using a 1D natural cubic spline [8] to use information from all points in the circular arc containing the unassigned pixel, rather than just its immediate neighbours.

$3 D$ nearest neighbour: Each unassigned voxel was given a value equal to the value of the nearest sampled voxel in $3 \mathrm{D}$ space.

\subsection{Method of comparison}

A total of sixty $3 \mathrm{D}$ images were created (10 patients $\times 2$ cardiac phases $\times 3$ interpolation methods) each of dimension $728 \times 728 \times 512$ pixels, and one reconstructed LV short axis planar image $(728 \times 728$ pixels $)$ was extracted from each for further analysis. To each short axis image, a $7 \times 7$ truncated pyramid edge detection operator [9] was applied, followed by thresholding to produce a binary "edge" map. Binary erosion was then applied to remove noise. The number of remaining "edge" pixels was counted, and used for further analysis.

The number of edge pixels was used as the comparator between the three interpolation methods. Each of the three combinations of pairs of interpolation methods were compared using the method of Bland and Altman [10], to find the mean difference of the number of pixels in the edge map, and the confidence interval of the mean difference. 
Table 1. Number of pixels calculated to be near to an anatomical boundary (edge) of short axis LV images $(728 \times$ 728 pixels) reconstructed from 3D cardiac ultrasound scans by three different interpolation methods: 1D linear (1DL), 1D spline (1DS) and 3D nearest neighbour (3DNN).

\begin{tabular}{crrrr}
\hline \hline Patient & Phase & \multicolumn{3}{c}{ Interpolation method } \\
\cline { 3 - 5 } & & 1DL & 1DS & 3DNN \\
\hline 1 & ED & 6155 & 5841 & 4626 \\
& ES & 5386 & 5305 & 4225 \\
2 & ED & 6474 & 5839 & 4712 \\
& ES & 6115 & 5330 & 4577 \\
3 & ED & 3758 & 3233 & 2719 \\
& ES & 3923 & 3494 & 3219 \\
4 & ED & 7904 & 7276 & 6566 \\
& ES & 6159 & 5694 & 4777 \\
5 & ED & 5315 & 4645 & 3862 \\
& ES & 5262 & 4855 & 3995 \\
6 & ED & 4472 & 4371 & 3659 \\
& ES & 4473 & 3893 & 3318 \\
7 & ED & 4107 & 3461 & 3281 \\
& ES & 4985 & 4479 & 3890 \\
8 & ED & 4860 & 4592 & 3984 \\
& ES & 4973 & 4550 & 4125 \\
9 & ED & 5454 & 4701 & 4183 \\
& ES & 4620 & 4213 & 3751 \\
10 & ED & 5387 & 5316 & 4052 \\
& ES & 4866 & 4330 & 3956 \\
\hline \hline mean & & 5232 & 4771 & 4074 \\
sd & & 983 & 964 & 790 \\
\hline \hline
\end{tabular}

\section{Results}

Figure 2 shows a reconstructed LV short axis image and corresponding binary edge map for patient 2 , using three methods of interpolation. There are visually perceptible differences between the reconstructed images for different interpolation methods, and these differences are also detectable in the corresponding edge maps. Table 1 shows the number of pixels calculated to be near to an anatomical boundary for 20 short axis LV images each reconstructed using three different interpolation methods. For the 1D linear method, the mean number of edge pixels was $0.99 \pm 0.19 \%$ image area, for the $1 \mathrm{D}$ spline method it was $0.90 \pm 0.18 \%$ image area, and for the 3D nearest neighbour method it was $0.77 \pm 0.15 \%$ image area.

Table 2 shows that the different methods calculate significantly different numbers of edge pixels. The differences in the number of edge pixels and their $95 \%$ confidence intervals are shown for each of the three pairs of comparisons of methods.
Table 2. Differences between the number of pixels calculated to lie near an anatomical boundary between three methods of voxel interpolation. The method of Bland and Altman was used to compare methods for the results given in table 1. All differences are significant at $95 \%$ confidence level.

\begin{tabular}{crr}
\hline \hline Methods & $\begin{array}{r}\text { Difference } \\
\text { (pixels) }\end{array}$ & $\begin{array}{r}95 \% \text { CI of difference } \\
\text { (pixels) }\end{array}$ \\
\hline 1DL-1DS & 467 & $368-565$ \\
1DL-3DNN & 1164 & $1025-1302$ \\
1DS-3DNN & 697 & $554-840$ \\
\hline \hline
\end{tabular}
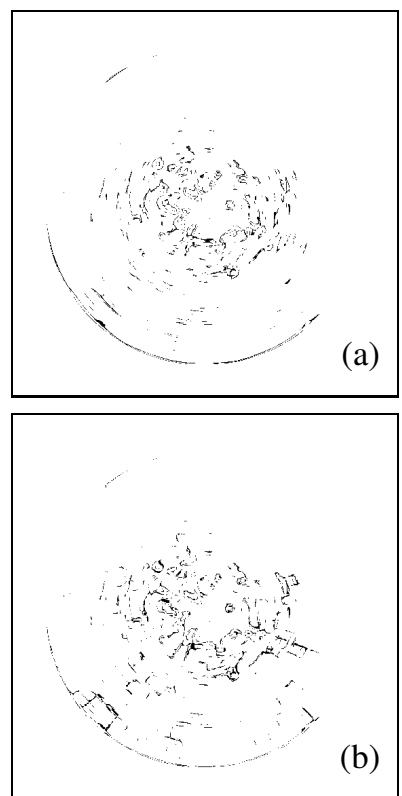

(b)

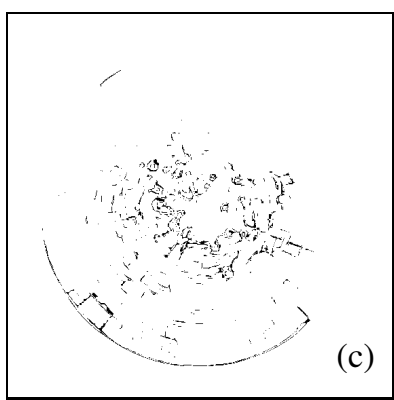

Figure 3. Comparison of pairs of binary edge maps of figure 2. The set of pixels are those which have been calculated to lie near to an edge in images created with one or other interpolation method, but not both:- (a) If $1 D L$ is the set of edge pixels arising from 1D linear interpolation and $1 D S$ the set from 1D spline interpolation, the set of pixels shown is $\{1 D L \cup 1 D S\} \backslash\{1 D L \cap 1 D S\}$; (b) $\{1 D L \cup 3 D N N\} \backslash\{1 D L \cap 3 D N N\}$ where $3 D N N$ is the set of edge pixels from 3D nearest neighbour interpolation; (c) $\{1 D S \cup 3 D N N\} \backslash\{1 D S \cap 3 D N N\}$. 


\section{Discussion}

Figure 3 illustrates, in part, the reason for differences between the different techniques. This figure shows the set of pixels which were calculated to lie near an edge in images created with one or other interpolation method, but not both, when two methods were compared. Strictly, if $A$ was the set of edge pixels for a reconstructed 2D image created with interpolation method $\mathrm{A}$, and $B$ was the set for interpolation method $\mathrm{B}$, then the images show the sets $\{A \cup B\} \backslash\{A \cap B\}$ for each of the three paired comparisons. Figure 3 part (a), shows the comparison of 1D linear and 1D spline interpolations. Differences arose (see parts (b) and (d) of figure 2) because spline interpolation tended to broaden edges whilst linear interpolation based only on neighbouring points tended to introduce small artefactual edges due to localised noise such as speckle. Parts (b) and (c) show the differences between 3D nearest neighbour interpolation and each of the two 1D methods. The majority of these difference pixels arose from the "blockiness" inherent in a nearest neighbour technique.

Our results suggest that spline-based interpolation is preferable to linear methods because of its ability to reject speckle noise. Nearest neighbour techniques introduce artefactual edges. In 3D, it is preferable to use information from all neighbouring voxels, rather than just those neighbours which lie in the plane of acquisition. We are currently investigating the use of spline methods in a $3 \mathrm{D}$ space.

The results show that the choice of interpolation technique employed during 3D ultrasound scan conversion has a significant effect on the results of quantitative image analysis techniques. Care must be taken to avoid the introduction of artefact, whether visual or quantitative, into $3 \mathrm{D}$ image analysis by poor choice of interpolation algorithm.

\section{References}

[1] Sims AJ, Pemberton J, Irvine T, Kenny A, Murray A. Mapping the left ventricular cavity by analysis of images reconstructed from 3D trans-oesophageal echocardiography. Computers in Cardiology 2002;29:351-354.

[2] Parker JA, Kenyon RV, Troxel DE. Comparison of interpolating methods for image resampling. IEEE Transactions on Medical Imaging 1983;MI-2:31-39.

[3] Maeland E. On the comparison of interpolation methods. IEEE Transactions on Medical Imaging 1988;7:213-217.

[4] Lehmann TM, Gönner C, Spitzer K. Survey: interpolation methods in medical image processing. IEEE Transactions on Medical Imaging 1999;18:1049-1075.

[5] Grevera GJ, Udupa JK. An objective comparison of 3-D image interpolation methods. IEEE Transactions on Medical Imaging 1998;17:642-652.

[6] Ostuni JL, Santha AKS, Mattay VS, Weinberger DR, Levin $\mathrm{RL}$, et al. Analysis of interpolation effects in the reslicing of functional MR images. Journal of Computer Assisted Tomography 1997;21:803-810.

[7] Pluim JPW, Maintz JBA, Viergever MA. Interpolation artefacts in mutual information-based image registration. Computer Vision and Image Understanding 2000;77:211232.

[8] Press WH, Teukolsky SA, Vetterling WT, Flannery BP. Numerical recipes in $\mathrm{C}$ : The art of scientific computing. Second edition. Cambridge, UK: Cambridge University Press, 1998.

[9] Pratt W. Digital Image Processing. Second edition. New York, USA: John Wiley \& Sons Inc, 1991.

[10] Bland JM, Altman D. Statistical methods for assessing agreement between two methods of clinical measurement. Lancet 1986;1 (8476):307-310.

Address for correspondence:

Andrew J Sims

Regional Medical Physics Department, Freeman Hospital Newcastle upon Tyne, UK.

tel.: +441912843111 ×26598

a.j.sims@newcastle.ac.uk 